\title{
Sequential replication-coupled destruction at G1/S ensures genome stability
}

\author{
Kate E. Coleman, ${ }^{1}$ Gavin D. Grant ${ }^{2}$ Rachel A. Haggerty, ${ }^{3,4}$ Kristen Brantley, ${ }^{2}$ Etsuko Shibata, ${ }^{5}$ \\ Benjamin D. Workman, ${ }^{2}$ Anindya Dutta, ${ }^{5}$ Dileep Varma, ${ }^{6}$ Jeremy E. Purvis, ${ }^{3,4}$ \\ and Jeanette Gowen Cook $^{1,2}$ \\ ${ }^{1}$ Curriculum in Genetics and Molecular Biology, The University of North Carolina at Chapel Hill, Chapel Hill, North Carolina \\ 27599, USA; ${ }^{2}$ Department of Biochemistry and Biophysics, The University of North Carolina at Chapel Hill, Chapel Hill, North \\ Carolina 27599, USA; ${ }^{3}$ Department of Genetics, The University of North Carolina at Chapel Hill, Chapel Hill, North Carolina \\ 27599, USA $;{ }^{4}$ Curriculum in Bioinformatics and Computational Biology, The University of North Carolina at Chapel Hill, Chapel \\ Hill, North Carolina 27599, USA; ${ }^{5}$ Department of Biochemistry and Molecular Genetics, University of Virginia School of \\ Medicine, Charlottesville, Virginia 22908, USA; ${ }^{6}$ Department of Cell and Molecular Biology, Northwestern University Feinberg \\ School of Medicine, Chicago, Illinois 60611, USA
}

Timely ubiquitin-mediated protein degradation is fundamental to cell cycle control, but the precise degradation order at each cell cycle phase transition is still unclear. We investigated the degradation order among substrates of a single human E3 ubiquitin ligase, CRL4 ${ }^{\text {Cdt2 }}$, which mediates the S-phase degradation of key cell cycle proteins, including Cdt1, PR-Set7, and p21. Our analysis of synchronized cells and asynchronously proliferating live single cells revealed a consistent order of replication-coupled destruction during both S-phase entry and DNA repair; Cdt1 is destroyed first, whereas $\mathbf{2} 21$ destruction is always substantially later than that of Cdt1. These differences are attributable to the CRL4 ${ }^{\mathrm{Cdt} 2}$ targeting motif known as the PIP degron, which binds DNA-loaded proliferating cell nuclear antigen $\left(\mathrm{PCNA}^{\mathrm{DNA}}\right)$ and recruits CRL4 ${ }^{\mathrm{Cdt} 2}$. Fusing Cdt1's PIP degron to $\mathrm{p} 21$ causes 21 to be destroyed nearly concurrently with Cdt1 rather than consecutively. This accelerated degradation conferred by the Cdt1 PIP degron is accompanied by more effective Cdt2 recruitment by Cdt1 even though p21 has higher affinity for PCNA $^{\text {DNA }}$. Importantly, cells with artificially accelerated $\mathrm{p} 21$ degradation display evidence of stalled replication in mid-S phase and sensitivity to replication arrest. We therefore propose that sequential degradation ensures orderly S-phase progression to avoid replication stress and genome instability.

[Keywords: replication stress; Cdt1; p21; CDK; ubiquitin; S phase]

Supplemental material is available for this article.

Received April 14, 2015; revised version accepted July 23, 2015.

A key regulatory mechanism ensuring unidirectional progression through the eukaryotic cell division cycle is the timely destruction of proteins via the ubiquitin-proteasome pathway. The precise control of ubiquitin-mediated proteolysis is critical to many cellular processes, including transcription, DNA replication, DNA repair, and chromosome segregation. Deregulated protein ubiquitylation can promote aberrant cell proliferation and genome instability (for review, see Nakayama and Nakayama 2006; Teixeira and Reed 2013). Ubiquitin-mediated degradation requires the selection of a protein substrate by an E3 ubiquitin ligase. Several E3 enzymes recognize their targets via an amino acid-binding motif in the substrate called a "degron." For the cullin family of E3 ligases, degron binding facilitates ubiquitin transfer from an associated E2 enzyme to the substrate; successive ubiquitylations direct ultimate degradation by the $26 \mathrm{~S}$ proteasome (Petroski and Deshaies 2005; Nakayama and Nakayama 2006).

Corresponding author: jgcook@email.unc.edu

Article published online ahead of print. Article and publication date are online at http://www.genesdev.org/cgi/doi/10.1101/gad.263731.115.
Selectivity and timing of E3 ligase action by regulating degron recognition or E3 ligase activity allow oscillations in protein abundance to control key events.

It is generally presumed that an individual E3 ubiquitin ligase targets its cohort of substrates simultaneously, leading to their near-synchronous degradation. Countering this notion, however, several substrates of the anaphase-promoting complex/cyclosome $(\mathrm{APC} / \mathrm{C})$, the E3 ubiquitin ligase complex regulating mitotic progression, are degraded sequentially. Mammalian APC/C ${ }^{\mathrm{Cdc} 20}$ triggers the degradation of substrates such as Nek2A and $\mathrm{Cy}$ clin A in prometaphase, well before the destruction of other APC/C substrates like securin and Cyclin B, which occurs during metaphase (Elzen and Pines 2001; Hagting et al. 2002). The mechanism distinguishing early versus late substrates involves spindle assembly checkpoint-

(C) 2015 Coleman et al. This article is distributed exclusively by Cold Spring Harbor Laboratory Press for the first six months after the full-issue publication date (see http://genesdev.cshlp.org/site/misc/terms. xhtml). After six months, it is available under a Creative Commons License (Attribution-NonCommercial 4.0 International), as described at http://creativecommons.org/licenses/by-nc/4.0/. 
driven changes in how the Cdc20 substrate adapter interacts with APC (Izawa and Pines 2011). Likewise, budding yeast APC/C directs the degradation of the mitotic cyclin Clb5 before securin; in this instance, the distinguishing mechanism involves cyclin-dependent kinase 1 (Cdk1)mediated substrate phosphorylation near a Cdc20-binding motif (Lu et al. 2014). The ordered degradation of these mitotic proteins is essential for coordinated and accurate mitotic progression and cytokinesis (Elzen and Pines 2001; Lindon and Pines 2004; Izawa and Pines 2011; Lu et al. 2014). It is not yet known whether similar patterns of degradation occur at other cell cycle transitions. In particular, the temporal regulation of protein degradation during S-phase entry is still poorly understood. We hypothesized that the events of S-phase onset must be highly temporally controlled to properly coordinate replication onset, S-phase progression, and precise genome duplication.

To test this idea, we investigated substrates of the CRL4 ${ }^{\text {Cdt2 }}$ E3 ubiquitin ligase, which triggers replicationcoupled destruction of several lynchpin cell cycle proteins during $\mathrm{S}$ phase. In human cells, these substrates include Cdt1 (Arias and Walter 2005; Nishitani et al. 2006; Senga et al. 2006) and PR-Set7 (Abbas et al. 2010; Centore et al. 2010; Oda et al. 2010; Jørgensen et al. 2011), two factors critical to DNA replication origin licensing; the S-phase CDK inhibitor p21 (Abbas et al. 2008; Nishitani et al. 2008 ); the DNA polymerase $\delta$ subunit p12 (Terai et al. 2013; Zhang et al. 2013; Zhao et al. 2014); the base excision repair enzyme thymine DNA glycosylase (TDG) (Shi- bata et al. 2014; Slenn et al. 2014); and the nucleotide excision repair endonuclease xeroderma pigmentosum group G (XPG) (Han et al. 2014). Each of these proteins is degraded by the same general mechanism, which involves DNA loading of the sliding clamp processivity factor proliferating cell nuclear antigen (PCNA) during DNA synthesis followed by binding of the substrate to DNAloaded PCNA (PCNA ${ }^{\mathrm{DNA}}$ ) via a PCNA-interacting motif known as a PIP box (Fig. 1A; Arias and Walter 2006; Senga et al. 2006). PIP boxes that confer replication-coupled destruction reside in specialized motifs termed PIP degrons that include additional residues necessary for recognition by CRL4 ${ }^{\text {Cdt2 }}$ (Havens and Walter 2009). The Cdt2 substrate adaptor subunit of CRL4 ${ }^{\text {Cdt2 }}$ recognizes a presumed composite surface consisting of the substrate PIP degron and specific residues from PCNA ${ }^{\mathrm{DNA}}$ (Arias and Walter 2006; Havens and Walter 2009; Havens et al. 2012). Importantly, Cdt2 cannot stimulate substrate ubiquitylation unless the substrate is first bound to PCNA ${ }^{\mathrm{DNA}}$, a requirement that couples substrate destruction directly to DNA synthesis (Havens and Walter 2009). Perturbations to replication-coupled destruction have profound effects on S-phase progression and genome stability (Arias and Walter 2005, 2006; Abbas et al. 2010; Centore et al. 2010; Oda et al. 2010; Tardat et al. 2010; Terai et al. 2013; Zhang et al. 2013; Shibata et al. 2014).

The cell cycle functions of CRL4 ${ }^{\text {Cdt2 }}$ substrates vary widely, raising the possibility that they may need to be degraded at different times relative to one another to ensure
A

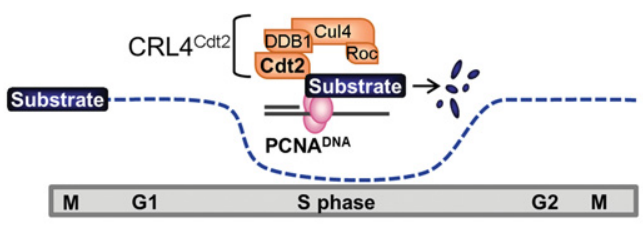

B

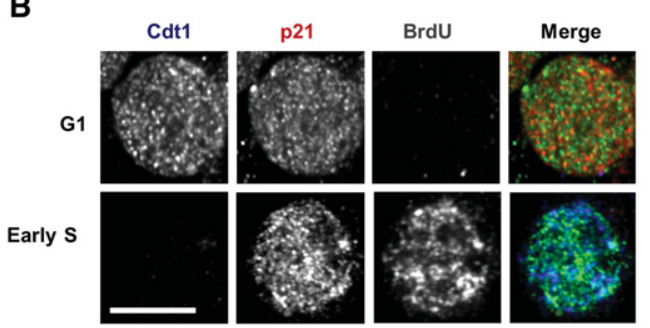

C

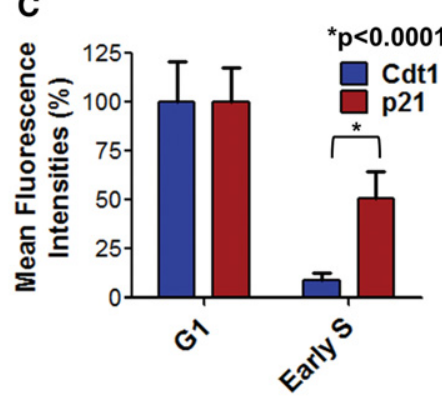

D

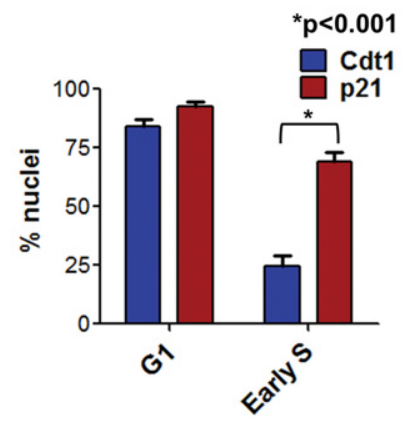

E

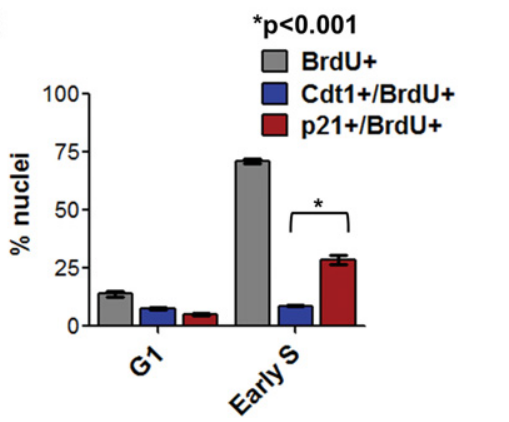

Figure 1. CRL4 $4^{\mathrm{Cdt} 2}$ substrates are degraded in a stereotypical order during early $\mathrm{S}$ phase. $(A)$ The mechanism of cell cycle regulation of CRL4 ${ }^{\text {Cdt2 }}$ substrates requires PCNA DNA loading. (B) HCT116 cells were synchronized by sequential thymidine and nocodazole treatment and fixed at $2.5 \mathrm{~h}$ (G1) or $4 \mathrm{~h}$ (early S) after nocodazole release. Cells were labeled with bromodeoxyuridine (BrdU) 30 min prior to fixation and staining with the indicated antibodies. Bar, $5 \mu \mathrm{m} .(C)$ Average fluorescence intensities relative to G1 from three independent experiments. $n=15$. $(D)$ Percentage of nuclei positively stained by anti-Cdt 1 or anti-p21. $n=650$. (E) Percentage of nuclei positively staining with the indicated antibodies from three independent experiments. $n=200$. Error bars indicate standard deviations. 
orderly transition from G1 to S phase. Although the molecular mechanism of CRL4 ${ }^{\mathrm{Cdt} 2}$-mediated degradation is well characterized, it is still unclear whether all substrates are degraded with similar timing. It is also unclear whether the relative timing of substrate destruction is important for the proper coordination of S-phase progression. In this study, we provide the first direct evidence of ordered substrate degradation of CRL4 ${ }^{\text {Cdt2 }}$ substrates and show that the timing of substrate degradation is related to differences in the substrates' PIP degron affinities for Cdt2. The sequential rather than simultaneous degradation pattern is essential to avoid replication stress during $\mathrm{S}$ phase.

\section{Results}

CRL4 $4^{\text {Cdt2 }}$ substrates are degraded sequentially during early $S$ phase

We first determined whether two CRL4 ${ }^{\text {Cdt2 }}$ substrates are degraded simultaneously or in a specified order at the G1/S transition. Toward that end, we quantified the change in Cdt1 and p21 concentrations in individual late G1-phase versus early S-phase cells. We synchronized HCT116 cells in mitosis by sequential thymidine and nocodazole blocks (Chandrasekaran et al. 2011), released them from nocodazole, and pulse-labeled them with bromodeoxyuridine (BrdU) prior to harvesting at time points corresponding to either G1 (2.5 h after release) or early S phase ( $4 \mathrm{~h}$ after release). We then immunostained these cells for endogenous Cdt1, p21, and BrdU incorporation. Both Cdt1 and p21 were readily detectable in G1, but, notably, Cdt1 was mostly degraded by early $S$ phase, whereas p21 was not (Fig. 1B); p21 was more fully degraded later in S (Supplemental Fig. 1) We quantified the mean nuclear fluorescence intensities (Fig. 1C) and scored nuclei for Cdt1 or p21 (Fig. 1D). In cells with detectable Cdt1 or p21, the concentration of Cdt 1 decreased $>10$-fold between G1 (2.5 h) and early S phase (4 h), but p21 levels decreased only twofold during that same time period (Fig. 1C). Furthermore, far fewer nuclei retained Cdt 1 at the early S-phase time point (24\%) than retained p21 (70\%). Moreover, we detected a relatively low occurrence of BrdU-positive cells that costained for Cdt1 (9\%), whereas BrdU/p21 double-positive cells were much more prevalent $30 \%$ of the total and nearly half of the BrdU-positive cells), suggesting that cells initiate DNA replication in the presence of p21 (Fig. 1E). We note that, at this single time point after synchronization, not all cells have yet entered S-phase, which contributes to the Cdt1-positive cells in Figure 1D.

A potential caveat to these immunofluorescence experiments was that the detection of endogenous Cdt1 and p21 required the use of different antibodies. To directly compare the rates of $\mathrm{Cdt} 1$ and $\mathrm{p} 21$ degradation using the same detection method, we generated fluorescent fusions. We fused cyan fluorescent protein (CFP; "mCerulean3") to the $\mathrm{C}$ terminus of $\mathrm{Cdt} 1$ and yellow fluorescent protein (YFP; "Venus") to the $\mathrm{N}$ terminus of $\mathrm{p} 21$ bearing a nuclear localization signal (NLS); these arrangements leave the regions of each protein that direct replication-coupled destruction unperturbed. We expressed them from a single bicistronic mRNA in which the two fusions were separated by a viral self-cleaving sequence from porcine teschovirus "P2A" (Fig. 2A; Kim et al. 2011). We integrated the bicistronic cassette into U2OS cells at a single FRT locus under control of a doxycycline-inducible promoter; the parent cells were engineered to constitutively express a histone H2B-mCherry fusion. Addition of doxycycline to the culture medium resulted in a dose-dependent induction of both fusions, and, importantly, no uncleaved protein was detectable even at the highest concentrations (Fig. 2A). Induction with $20 \mathrm{ng} / \mathrm{mL}$ doxycycline produced YFP-p21 at close to endogenous levels, and Cdt1-CFP was moderately overproduced, so we selected this concentration for subsequent experiments (Fig. 2A, lane 3). Importantly, both fusions showed the same stability as their endogenous counterparts (Supplemental Fig. 2A,B), and much higher levels of expression were required to produce detectable cell cycle perturbations (Supplemental Fig. 2C-E). Based on our immunostaining results (Fig. 1), we predicted that Cdt1-CFP would be consistently degraded before YFP-p21 and that, in asynchronous populations, we would observe YFP single-positive cells but few CFP single-positive cells. To test this prediction, we imaged fixed asynchronous, unperturbed cells by fluorescence microscopy (Fig. 2B). We identified nuclei and scored them as either YFP (p21)-positive, CFP (Cdt1)-positive, double-positive, or double-negative. Among cells with detectable fluorescence expression, double positives were the most abundant class, representing cells presumed to be in either G1 or G2 phase (31\%). (Of note, we did not achieve $100 \%$ expression under these induction conditions, meaning that we cannot conclude that all double-negative nuclei are in S phase.) Strikingly, however, YFP-p21 single positives represented a significant subpopulation $(22 \%)$, whereas Cdt1-CFP single positives were exceedingly rare $(0.3 \%)$ (Fig. 2C). Altogether, these results suggest that the CRL4 ${ }^{\mathrm{Cdt} 2}$ substrates Cdt 1 and p 21 undergo sequential rather than simultaneous degradation upon S-phase entry during an unperturbed cell cycle. This finding prompted investigation into the molecular mechanism of this differential targeting.

\section{CRL4 ${ }^{\text {Cdt2 }}$ substrates are degraded sequentially during DNA repair synthesis}

Since most DNA repair processes involve a DNA synthesis step, PCNA is loaded during DNA repair, similar to its DNA loading during S phase. Consequently, CRL4 ${ }^{\text {Cdt2 }}$ substrates are also degraded during DNA repair (Senga et al. 2006; Abbas et al. 2008, 2010; Nishitani et al. 2008 ; ). We took advantage of this knowledge to induce synchronous PCNA loading and therefore simultaneous and robust replication-coupled destruction in otherwise asynchronous populations. We employed a semiquantitative immunoblotting strategy to compare the relative degradation kinetics during DNA repair for CRL4 ${ }^{\text {Cdt2 }}$ substrates (Materials and Methods; Supplemental Fig. 3A). To determine whether CRL4 ${ }^{\mathrm{Cdt} 2}$-mediated destruction also occurs sequentially during DNA repair, we irradiated HCT116 cells with $20 \mathrm{~J} / \mathrm{m}^{2} \mathrm{UV}$ and monitored levels of 

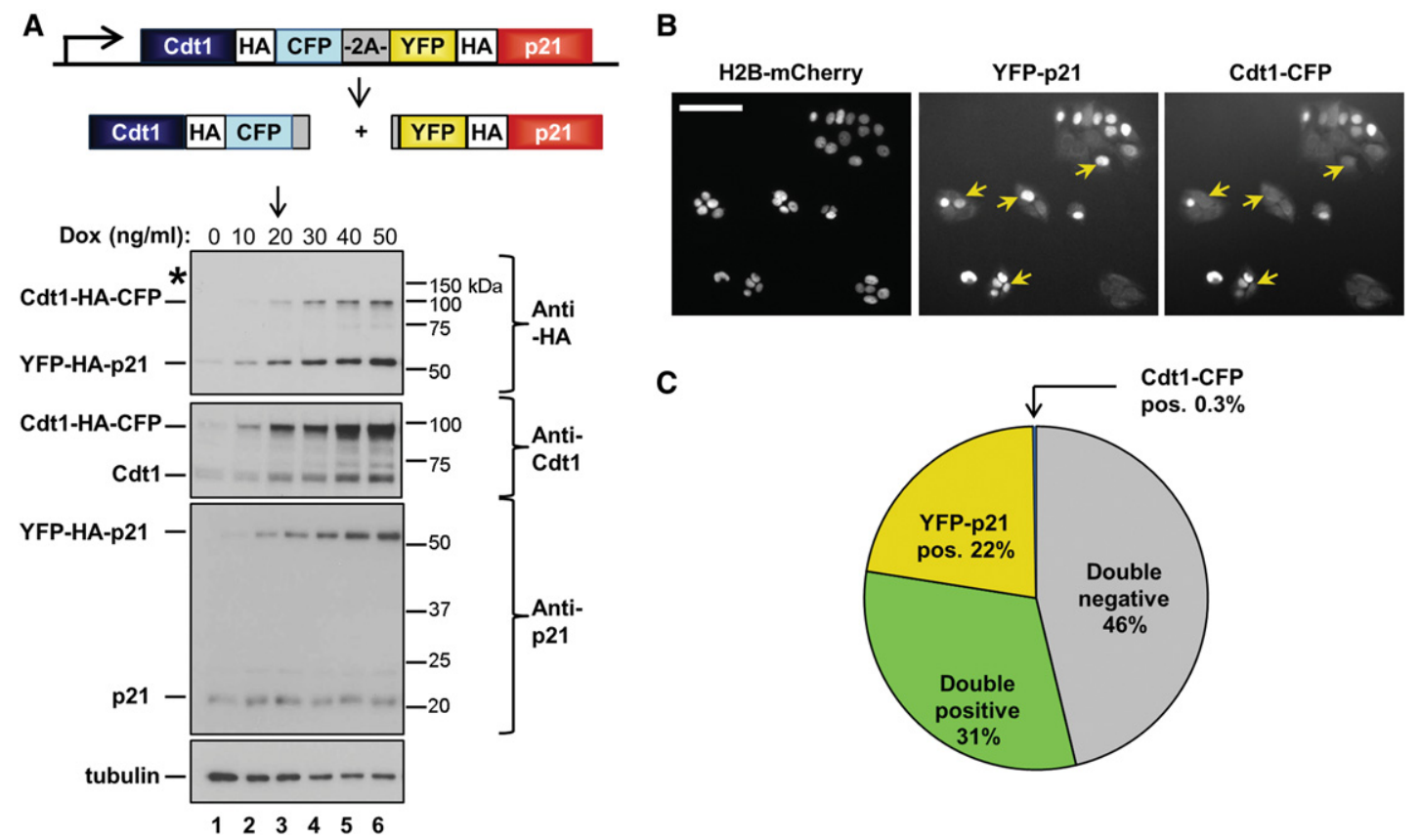

C

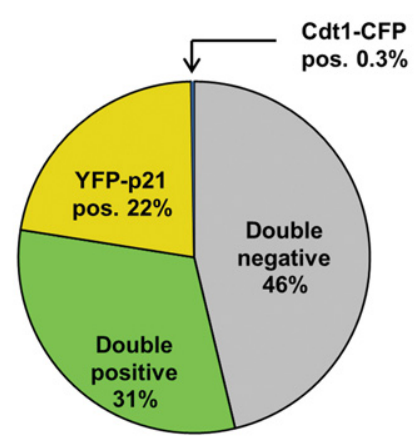

Figure 2. Cdt1-CFP is degraded more rapidly than YFP-p21 in unperturbed cells. $(A$, top) Schematic of the bicistronic expression construct; Cdt1-HA-mCerulean3 (CFP) and Venus (YFP)-HA-p21 are produced from a single mRNA due to the presence of a viral self-cleaving peptide, "2A." (Bottom) The resulting Cdt1 and p21 fusions were expressed from a single FRT locus in U2OS cells after doxycycline induction with the indicated concentrations for $20 \mathrm{~h}$. The arrow marks the concentration used for imaging, and the asterisk marks the predicted size of the fusion in the absence of 2A-mediated cleavage. (B) A representative field showing double-negative, double-positive, and YFP (Venus) single-positive cells; four single-positive cells are marked with arrowheads. Bar, $100 \mu M$. (C) Quantification of asynchronous Cdt1-mCerulean3-expressing and Venus-p21expressing U2OS cells. Averages from three fields of cells $(n>150$ per field $)$ were scored as double-negative, double-positive, or single-positive.

three CRL4 ${ }^{\text {Cdt2 }}$ targets-Cdt1, PR-Set7, and p21-in a time course. Similar to our analysis of the G1/S transition, Cdt1 was degraded dramatically faster than p21. By 15 min post-irradiation, Cdt 1 was already $>50 \%$ degraded, but p 21 persisted much longer and was not substantially degraded until 60-120 min post-irradiation (Fig. 3A, cf. lanes 2,5 and lane 1). The half-life of Cdt1 was only 9 min, but p21 was degraded with an average half-life of $53 \mathrm{~min}$; the half-time for PR-Set7 degradation was intermediate at $23 \mathrm{~min}$ (Fig. 3A). Importantly, we observed the same sequential relationship among the CRL4 ${ }^{\mathrm{Cdt} 2}$ substrates using other damaging agents such as oxidative damage (Fig. 3B) and the radiomimetic bleocin (data not shown). Moreover, the degradation order was similar in other cell lines, including immortalized primary fibroblasts (NHF-hTERT) and U2OS cells (Fig. 3C).

We also observed the same relative degradation order in cycloheximide-treated cells (Supplemental Fig. 3B) and that p21 stably expressed from a heterologous promoter was degraded with the same kinetics as endogenous p21 (Fig. 4A; Supplemental Fig. 3B). These observations eliminated the possibility that the particularly slow p21 degradation reflected a contribution from p53-dependent p21 induction. We note that $p 21$ is a well-known DNA damage-inducible gene, making the degradation of $\mathrm{p} 21$ protein after DNA damage somewhat counterintuitive. p21 induction and cell cycle arrest typically occur at lower doses and much later time points after DNA damage (Pagano et al. 1994; Bendjennat et al. 2003); in agreement with these previous studies, we also observed that p21 induction and cell cycle arrest only occurred $24 \mathrm{~h}$ after we treated HCT1 16 cells with $2-5 \mathrm{~J} / \mathrm{m}^{2} \mathrm{UV}$ (Supplemental Fig. 3C, D). On the other hand, CRL4 ${ }^{\mathrm{Cdt} 2}$-mediated p 21 destruction within 2-3 h was the predominant response to the higher doses of UV $\left(20 \mathrm{~J} / \mathrm{m}^{2}\right)$ used throughout this study. HeLa cells that are functionally p53-deficient also degraded Cdt1 more rapidly than they degraded PR-Set7 (Supplemental Fig. 3E). A recent study implicated the tripartite motif 39 (TRIM39) protein in regulating p21 degradation by blocking Cdt 2 binding and inhibiting p 21 degradation (Zhang et al. 2012). We tested a mutant form of p21 that fails to bind TRIM39 described in that study (p21$\mathrm{K} 153 \mathrm{~A})$ but observed no appreciable acceleration in its rate of degradation compared with the wild-type version (Supplemental Fig. 3F). Thus, we determined that slow p21 degradation is common to many human cell lines and must be via a mechanism unrelated to de novo protein synthesis or interaction with TRIM39.

\section{The Cdt1 PIP degron confers an accelerated degradation rate}

Human Cdt1 codons 1-28 encode its single PIP degron, and, when fused to a heterologous substrate, this sequence is sufficient to confer CRL4 ${ }^{\mathrm{Cdt} 2}$-mediated degradation (Senga et al. 2006; Rizzardi et al. 2014). Given our finding 
A

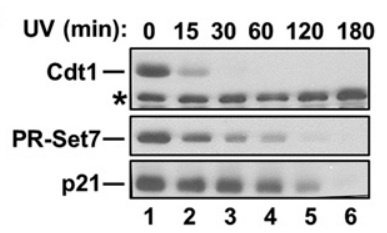

B

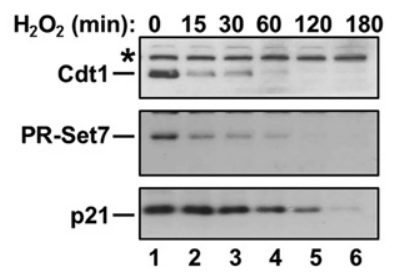

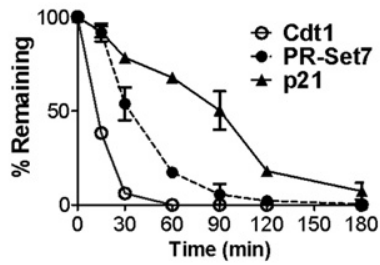

C

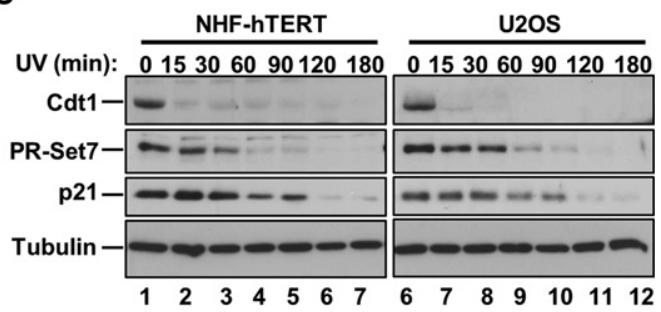

Figure 3. CRL4 ${ }^{\mathrm{Cdt} 2}$ substrates are degraded sequentially during DNA repair synthesis. HCT116 cells were treated with $20 \mathrm{~J} / \mathrm{m}^{2} \mathrm{UV}$ $(A)$ or $100 \mu \mathrm{M}$ tert-butyl peroxide $(B)$ and harvested at the indicated times after treatment for immunoblot analysis. Nonspecific bands serving as loading controls are indicated by asterisks. Quantifications of immunoblots from a minimum of three biological replicates of the UV time course are plotted. $(C)$ NHFhTERT cells (lanes 1-7) or U2OS cells (lanes 8-14) were treated with $20 \mathrm{~J} / \mathrm{m}^{2} \mathrm{UV}$ and harvested at the indicated time points as in $A$. Error bars indicate standard error of the mean (SEM). that $\mathrm{Cdt} 1$ is consistently the most rapidly degraded among the CRL4 ${ }^{\text {Cdt2 }}$ substrates tested, we sought to determine whether the Cdt1 PIP degron is sufficient to confer the fast kinetics of degradation. We therefore fused Cdt1 128 to the $\mathrm{N}$ terminus of an epitope-tagged p21 and compared this fusion with a correspondingly epitope-tagged wild-type $\mathrm{p} 21$. We generated stable HCT116 cell lines producing these fusions at near-endogenous levels and used the same UV irradiation strategy to induce synchronous replication-coupled destruction as in Figure 3. First, the addition of an N-terminal HA epitope tag to p21 had no measureable effect on degradation kinetics; both endogenous and tagged p21 were degraded at the same rate and substantially slower than endogenous Cdt1 (Fig. 4A). Second, constitutive expression of HA-p21 from a heterologous promoter had no effect on $\mathrm{p} 21$ degradation kinetics (Fig. 4A; Supplemental Fig. 3B) and did not alter cell cycle phase distribution (Supplemental Fig. 3C [lane 6], D). Strikingly however, the PIP ${ }^{\mathrm{Cdt} 1}$-p21 chimera was degraded much more rapidly than either wild-type p 21 or endogenous $\mathrm{p} 21$, and this chimeric protein more closely resembled the degradation kinetics of endogenous Cdt1 with a half-life of just $20 \mathrm{~min}$ (Fig. 4B). A PIP ${ }^{\mathrm{Cdt} 1}$-p21 chimera in which key amino acids of the Cdt1 PIP degron were mutated to alanine (PIPm ${ }^{\mathrm{Cdt1}}$-p21) was degraded with kinetics similar to both wild-type HA-p21 and endogenous p21, as expected (Fig. 4C). Thus, addition of the Cdt1 PIP degron to the $\mathrm{N}$ terminus of $\mathrm{p} 21$ is sufficient to confer accelerated p21 degradation.

We considered several explanations for the rapid degradation of the PIP ${ }^{\mathrm{Cdt} 1}-\mathrm{p} 21$ fusion: the addition of a second PIP degron, the N-terminal location of the PIP degron, or the PIP degron sequence itself. To test the idea that rapid degradation is achieved simply by the presence of two degrons, we disrupted the native p21 PIP degron sequence in the PIP ${ }^{\text {Cdt1 }}$-p21 chimera, leaving only the one Cdt1 PIP degron, "PIPCdt1 -p21PIPm." We confirmed p21 PIP degron impairment in a control experiment (Supplemental Fig. $4 \mathrm{~A}, \mathrm{~B})$. The PIP ${ }^{\mathrm{Cdt1}}$-p21 PIPm fusion was still degraded faster than endogenous $\mathrm{p} 21$ (Fig. 4D), indicating that the presence of two degron sequences did not account for the accelerated degradation of the PIP ${ }^{\mathrm{Cdt} 1}$-p21 fusion in Figure 4B. The native PIP degron sequences of Cdt 1 and $\mathrm{p} 21$ are located at either the $\mathrm{N}$ or $\mathrm{C}$ terminus of each protein, respectively; thus, the relative location of the PIP degrons could impact accessibility to PCNA and/or CRL4 ${ }^{\mathrm{Cdt} 2}$. Importantly, when we fused a second copy of p21's PIP degron to its own $\mathrm{N}$ terminus, this $\mathrm{PIP}^{\mathrm{p} 21}-\mathrm{p} 21$ fusion was degraded slowly, like endogenous p21 (Fig. 4E). Finally, we fused the p21 PIP degron to an HA-tagged form of Cdt1 lacking its own native PIP degron. We confirmed that the tag did not disrupt wild-type Cdt1 degradation kinetics (Fig. 4F) and that deleting Cdt1's PIP degron stabilized Cdt1 independently (Supplemental Fig. 4C). Cdt1 bearing a single PIP degron derived from $\mathrm{p} 21$ was degraded more slowly than wild-type Cdt1, particularly at later time points (Fig. 4F). These experiments yielded several important mechanistic insights: The PIP degron sequence itself carries intrinsic and transferable information dictating the degradation kinetics, the location of the PIP degron sequence has little impact on degradation kinetics, and an additional PIP degron sequence is insufficient to enhance the degradation rate.

The accelerated degradation of the PIP ${ }^{\mathrm{Cdt}}-\mathrm{p} 21$ fusion during DNA repair suggested that a similar fusion would be degraded with faster kinetics in early S phase, since the same ubiquitylation pathway applies to both $S$ phase and DNA repair. To test that idea, we fused YFP to the $\mathrm{C}$ terminus of PIP ${ }^{\mathrm{Cdt} 1}$-containing $\mathrm{p} 21$ and integrated this doxycycline-inducible fusion into U2OS cells expressing histone H2B-mCherry. As a control, we integrated a YFP-p21 fusion to create a separate cell line. (The arrangement of these fusions was designed to avoid perturbing the relevant PIP degrons.) Of note, high concentrations of doxycycline induced substantial p21 overproduction and G1 accumulation, indicating that both fusions are likely active CDK inhibitors (Supplemental Fig. 5A,B), but cells can tolerate moderate p21 overproduction (e.g., from induction with $10-30 \mathrm{ng} / \mathrm{mL}$ ) with no detectable change in cell cycle phase distribution (Supplemental Fig. 5B). Moreover, the UV-triggered degradation of PIP $^{\text {Cdt1 }}$-p21-YFP was accelerated compared with normal 
A

в

C

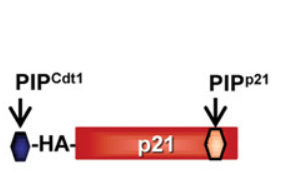

D

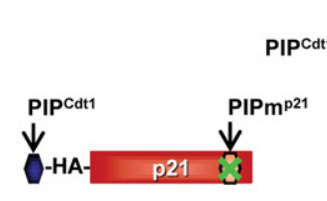

E

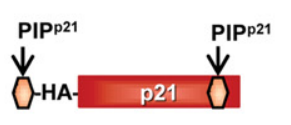

$\mathbf{F}$
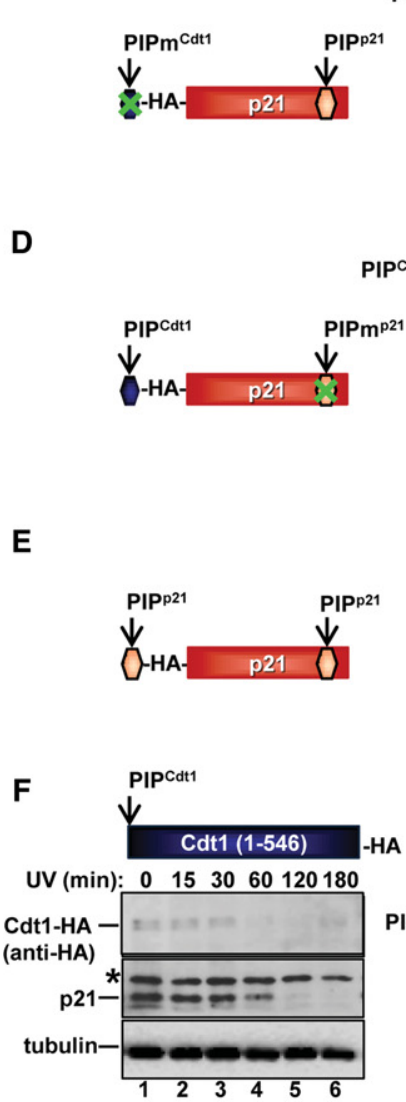
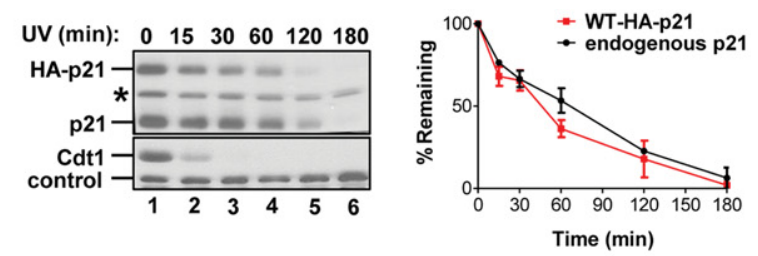

UV (min): 015306090120180
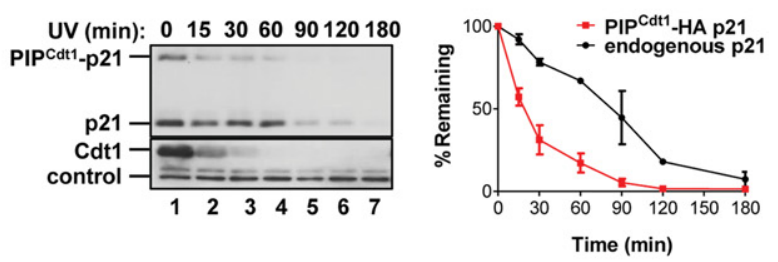

UV (min): 015306090120180
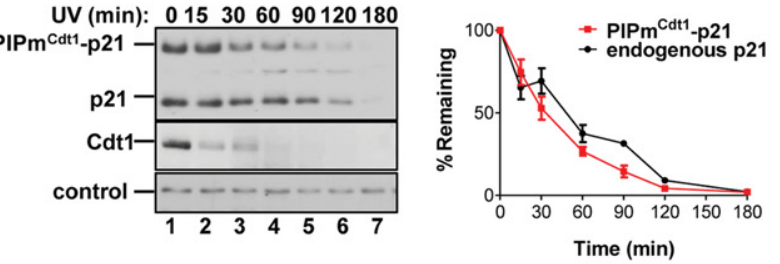

UV (min): 015306090120180
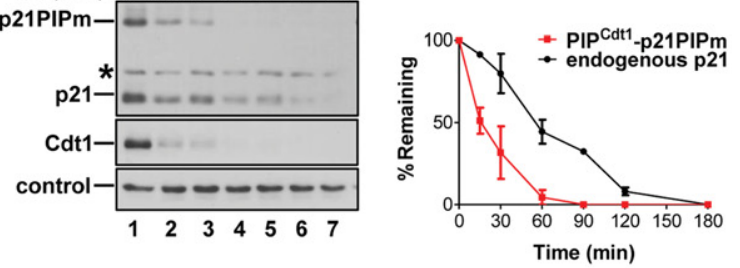

UV (min): 015306090120180

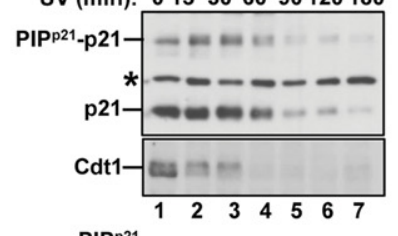

PIPp21

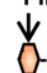

D-HA- $\Delta$ Cdt1 (15-546)

UV (min): $\begin{array}{lllll}0 & 15 & 30 & 60120180\end{array}$

PIPP21-HA-Cdt1

(anti-HA)

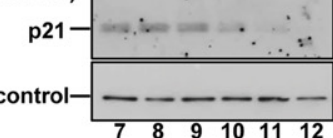

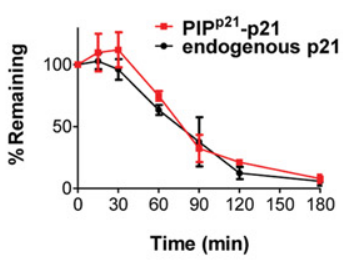

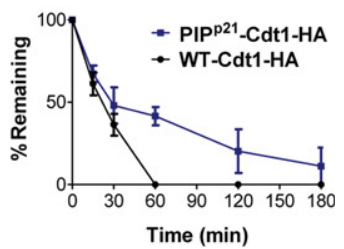

Figure 4. The Cdt1 PIP degron confers accelerated degradation to p21 during DNA repair. $(A)$ HCT116 cell lines were generated that stably produce HA-tagged wild-type p21 (A), HA-tagged PIP ${ }^{\text {Cdt1 }}$-p2 $1(B)$, HA-tagged PIPm ${ }^{\text {Cdt1 }}$-p21 bearing inactivating mutations in the Cdt1 PIP degron $(C)$, an HA-tagged PIP ${ }^{\text {Cdt1 }}$-p21 fusion with the native $\mathrm{p} 21$ PIP degron inactivated $(D)$, or PIP ${ }^{\mathrm{p} 21}$-p21 with the native p21 PIP degron added to the $\mathrm{N}$ terminus $(E)$. $(F)$ HA-tagged wild-type Cdt1 or Cdt1 lacking its native PIP degron (" $\Delta^{\prime \prime}$ ) but fused to the p21 PIP degron was expressed from an inducible promoter in U2OS cells with $20 \mathrm{ng} / \mathrm{mL}$ doxycycline. These cell populations were treated with $20 \mathrm{~J} / \mathrm{m}^{2} \mathrm{UV}$ and harvested at the indicated times for immunoblot analysis. Quantifications of immunoblots for ectopic and endogenous p21 and Cdt1 proteins are from two independent experiments. Error bars indicate SEM.

YFP-p21 or endogenous p21 similar to the HA-tagged fusions in Figure 4B (Supplemental Fig. 5C).

We induced expression of both $\mathrm{p} 21$ fusions to similar levels (10 ng/mL for wild-type and $30 \mathrm{ng} / \mathrm{mL}$ for PIP ${ }^{\mathrm{Cdt} 1}$. p21) and imaged unperturbed proliferating cells for at least $16 \mathrm{~h}$, capturing fluorescence images of histone H2B-
mCherry and YFP-p21 every $10 \mathrm{~min}$ (see representative Supplemental Movies 1,2). We subjected the videos to computational segmentation using the histone H2BmCherry to mark and track nuclei and then automatically quantified the average fluorescence intensity of YFP in both lines (see the Materials and Methods). Each set of 
fluorescence intensities was normalized from 0 (minimum) to 1 (maximum) for 50 cells that we selected for expression of the fusion and that underwent both mitosis and a complete degradation/reaccumulation cycle (i.e., $S$ phase) within the recorded time. The dynamics of YFP-p21 degradation are shown for these cells as a heat map in Figure 5A, with the first data point plotted for each cell corresponding to the video frame containing the cell division that produced that cell. Fitting these in silico aligned traces for each cell to an inverse sigmoidal curve yielded $T_{\mathrm{d}}$, the time from mitosis to the half-point of degradation (Fig. 5B). We then calculated the relative maximal rates of degradation from the slopes at each $T_{\mathrm{d}}$ and noted that the degradation rate of the PIPCdt1 -containing fusion was more than five times that of the fusion bearing only the native p21 PIP degron.

\section{Differential recruitment of PCNA/Cdt2 by PIP degrons}

We next generated new degron fusions in which either the Cdt1 or p21 PIP degron plus a NLS was fused to glutathione-S-transferase (GST). These fusions created an artificial substrate for replication-coupled CRL4 ${ }^{\text {Cdt2 }}$-mediated proteolysis and had the added benefit of isolating the Cdt1 and p21 PIP degrons from their respective full-length proteins (Fig. 6A). We monitored the degradation kinetics of the GST fusions after UV irradiation as before and found that PIP ${ }^{21}$-GST was degraded more slowly than PIPCdt1 GST, similar to the relationship between the endogenous proteins from which they were derived (Fig. 6A).

Two critical interactions for productive replicationcoupled destruction are binding of the PIP degron to PCNA $^{\text {DNA }}$ and subsequent recruitment of CRL4 through substrate interaction with the substrate receptor Cdt2. Differences in affinities for PCNA ${ }^{\text {DNA }}$ and/or Cdt 2 could explain why proteins containing the Cdt1 PIP degron are consistently degraded before proteins with the p21 PIP degron. To test this idea, we produced the same PIP ${ }^{\text {Cdt1 }}$ GST and PIP ${ }^{21}$-GST constructs in bacteria, isolated the recombinant proteins on glutathione beads, and used them to retrieve PCNA ${ }^{\mathrm{DNA}}$ and Cdt 2 from sonicated chromatin fractions of human cell lysates. We had previously used this strategy to analyze interactions of full-length Cdt1 with PCNA ${ }^{\text {DNA }}$ and Cdt2 (Chandrasekaran et al. 2011). As controls, we included a full-length GST-tagged p21 and a PIP mutant form of p21 that cannot bind PCNA, PIP mp21-GST (Rizzardi et al. 2014). As expected, the PIP mutant p21, unlike the wild-type p21, did not bind PCNA ${ }^{\text {DNA }}$ (Supplemental Fig. 6).

First, although equal amounts of solubilized chromatin were incubated with the two PIP degron fusions, the p21 PIP degron retrieved approximately three times more PCNA than the Cdt1 PIP degron did under the same conditions (Fig. 6B, cf. lanes 3 and 4). This tight binding of p21 to PCNA is consistent with prior studies (Gulbis et al. 1996; Arias and Walter 2006). Surprisingly, even with this strong relative binding to PCNA, the p21 PIP degron did not recruit proportionately more Cdt2 (Fig. 6B, cf. lanes 3 and 4). In fact, PIP ${ }^{\mathrm{Cdt} 1}$-GST retrieved more Cdt2 than PIP ${ }^{\mathrm{p} 21}-\mathrm{GST}$ did despite holding threefold less PCNA $^{\text {DNA }}$ (Fig. 6B, lanes 3,4). The significantly better Cdt2 binding with PIP ${ }^{\mathrm{Cdt} 1}$ GST was most readily apparent when we directly compared equal levels of copurified PCNA; PIP ${ }^{\text {Cdt1 }}$-GST bound more than fivefold more Cdt2 than PIP ${ }^{21}$-GST did for the same amount of copurified PCNA (Fig. 6C,D, lanes 3,4). Thus, the Cdt1 PIP degron more efficiently recruits $\mathrm{Cdt} 2$, and this effect likely contributes to its accelerated degradation via the CRL4 ${ }^{\text {Cdt2 }}$ E3 ubiquitin ligase.

We also considered a potential role for ubiquitin E2 utilization in the differential degradation of Cdt1 and p21. Ubiquitylation by cullin E3 ligases is catalyzed by
A

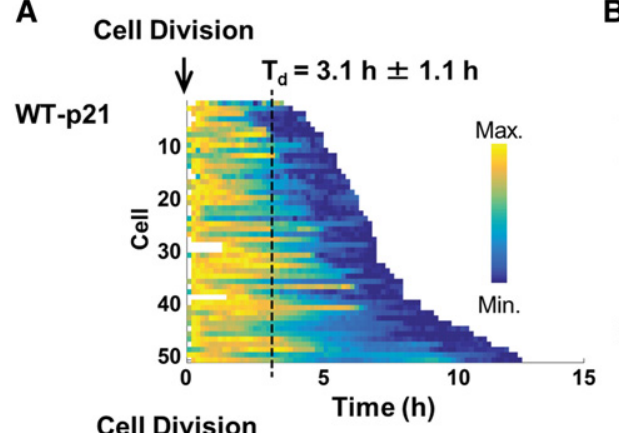

B
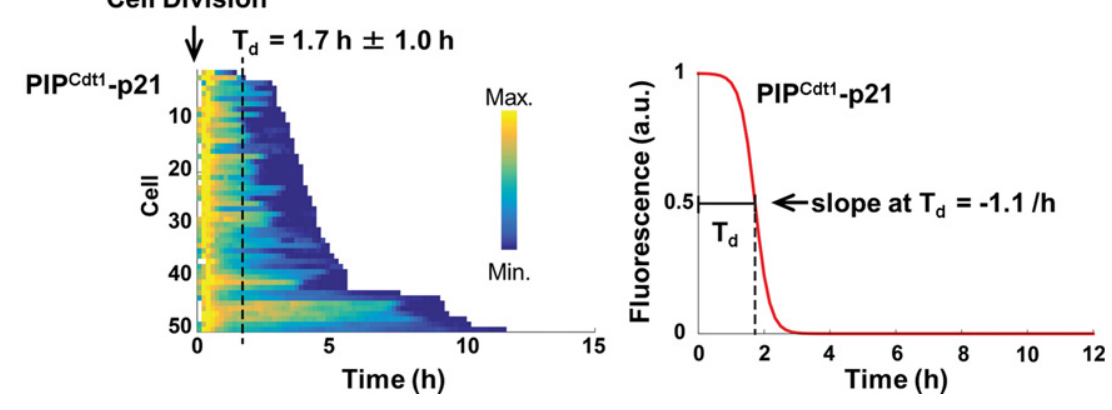

Figure 5. The Cdt1 PIP degron confers accelerated degradation to p21 at G1/S. (A) Heat maps for the kinetics of YFP-p21 and PIP ${ }^{\text {Cdt1 }}$. p21-YFP degradation in 50 asynchronously proliferating cells generated by high-content live-cell imaging. Expression was induced with $30 \mathrm{ng} / \mathrm{mL}$ doxycycline for wild-type YFP-p21 and $10 \mathrm{ng} / \mathrm{mL}$ doxycycline for PIP ${ }^{\mathrm{Cdt} 1}$-p21-YFP (see Supplemental Fig. 5), and individual cell fluorescence intensity traces were computationally aligned to mitosis $(t=$ 0 ). Fluorescence intensities were normalized to individual minima and maxima (see the Materials and Methods). (B) Data were fit to sigmoidal curves, and the average time for degradation from mitosis to the half minimum fluorescence intensity, $T_{\mathrm{d}}$, is marked with black dashed lines on both the heat maps and the plots. The slopes at $T_{\mathrm{d}}$ are indicated. 
A
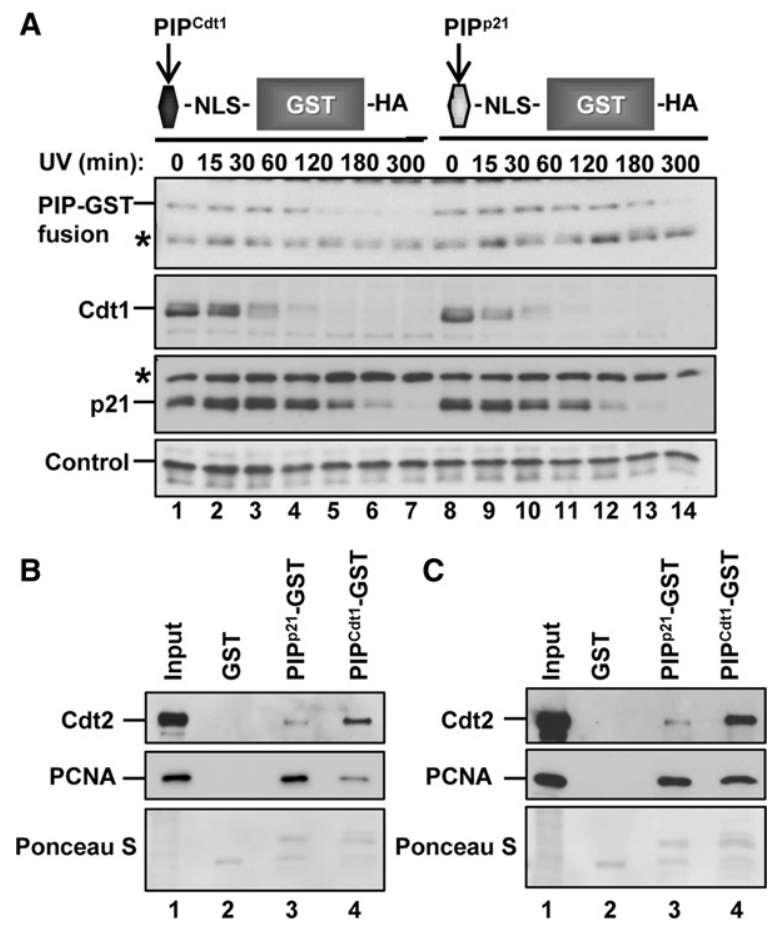

D

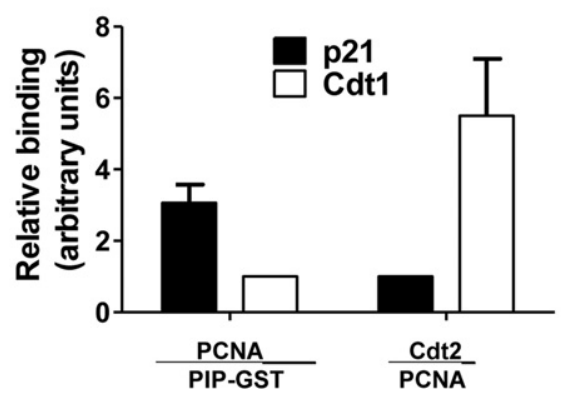

Figure 6. The Cdt1 PIP degron binds more Cdt 2 than the p 21 PIP degron. (A) HCT116 cells stably expressing either Cdt1 PIP (amino acids 1-28) or p21 PIP (amino acids 137-164) fused to the $\mathrm{N}$ terminus of GST were UV-treated and harvested for immunoblot analysis at the indicated times. (B) PIP-GST fusion proteins were purified from Escherichia coli lysates with glutathione-sepharose beads and incubated with sonicated chromatin fractions from UV-irradiated $293 \mathrm{~T}$ cells. Bound proteins were eluted in $2 \times$ SDS buffer and subjected to immunoblot analysis with the indicated antibodies. Lanes 2-4 contained equivalent amounts of GST fusions. (C) PIP degron-bound proteins were prepared as in $B$ but loaded equivalently for copurified PCNA. $(D)$ Quantification of the relative amounts of bound PCNA and $\mathrm{Cdt} 2$, based on two independent experiments. Error bars indicate standard deviations.

associated E2 ubiquitin-conjugating enzymes (UBCs), and our recent study showed that ubiquitylation of $\mathrm{p} 21$ and Cdt1 is carried out by different E2s: UBE2G1 + UBE2G2 for Cdt1 and UBCH8 for p21 (Shibata et al. 2011). Different E2s may be responsible for the different degradation rates, and, furthermore, exchanging the PIP degrons might have led to E2 switching. To test this hypothesis, we used RNAi-mediated depletion of the relevant E2s to determine whether the PIP ${ }^{\mathrm{Cdt} 1}$-p21 fusion (diagramed in Fig.
4B) relies on the E2 that normally targets Cdt1 (i.e., UBE2G1 + UBE2G2). As shown in Supplemental Figure 7, A and B, however, the PIPCdt1 - p21 fusion, just like endogenous p21, was stabilized in UBCH8-depleted cells, whereas its typical accelerated degradation pattern was observed in both control and UBE2G1 + UBE2G2-depleted cells. Thus, we concluded that degradation of PIP ${ }^{\mathrm{Cdt} 1}-\mathrm{p} 21$ still involves the same E2 as normal p21, arguing against an E2 switch. Substrate features separate from those directly responsible for E3 recruitment must control E2 specificity, but that discrimination plays little role in the rate of $\mathrm{CRL} 4{ }^{\mathrm{Cdt} 2}$-mediated degradation.

\section{Delayed p21 degradation facilitates normal S-phase progression}

We next sought to probe the physiological importance of sequential CRL4 ${ }^{\mathrm{Cdt} 2}$ substrate degradation. Specifically, we tested the consequences of imposing the early Cdt1 degradation pattern on p21. For this purpose, we stably produced siRNA-resistant versions of the PIP ${ }^{\mathrm{Cdt} 1}-\mathrm{p} 21$ and wild-type p 21 described in Figure 4 in HCT116 cells. We depleted endogenous p 21 and analyzed cell cycle profiles by flow cytometric measurement of BrdU incorporation and DNA content (Fig. 7A). Neither siRNA depletion of p21 nor stable expression of wild-type p21 had a substantial effect on overall cell cycle distribution in these cells (Fig. 7B), but we noted that p21 depletion caused an increase in a subpopulation of cells of intermediate DNA content (i.e., S phase) that were not actively synthesizing DNA. We scored this population as "BrdU neg/S phase," and, following p21 depletion, there were three times more $\mathrm{BrdU}^{\text {neg }} / \mathrm{S}$-phase cells compared with control cells. This phenotype was complemented by ectopic wildtype p21 (without causing cell cycle arrest), but, strikingly, PIP ${ }^{\mathrm{Cdt} 1}-\mathrm{p} 21$ cells still generated the $\mathrm{BrdU}^{\text {neg }} / \mathrm{S}$-phase subpopulation (Fig. 7A,B). PIP ${ }^{\mathrm{Cdt} 1}$-p21 was produced at levels similar to endogenous p21 in control RNA-treated cells, which suggests that the phenotype of the PIPCdt1 p21 line is the kinetics of p21 degradation rather than insufficient p21 (Fig. 7C).

The increase in cells that reached mid-S phase but then stopped DNA synthesis (BrdU ${ }^{\text {neg }} / \mathrm{S}$ phase) suggested that premature $\mathrm{p} 21$ degradation leads to difficulty in maintaining active replication. If true, then these cells may experience higher than normal rates of endogenous DNA damage, which could render them more sensitive to additional damage or replication stress. To test that idea more directly, we produced the same $\mathrm{p} 21$ fusions as YFP-tagged forms (i.e., Fig. 5) in U2OS cells and then challenged them to recover from a strong replication block. We depleted endogenous p21 (Supplemental Fig. 8A) and then held cells in hydroxyurea $(\mathrm{HU})$ for $16 \mathrm{~h}$ to induce replication stalling. We immunostained for a marker of DNA damage often associated with replication stress, $\gamma-\mathrm{H} 2 \mathrm{AX}$, and found the expected high percentage of positively staining cells in all HU-arrested populations (Fig. 7D,E). Releasing cells into fresh medium allowed S-phase resumption within $12 \mathrm{~h}$ in cells expressing normal p21 as measured by both flow cytometry (Supplemental Fig. 8B) and elimination 
A

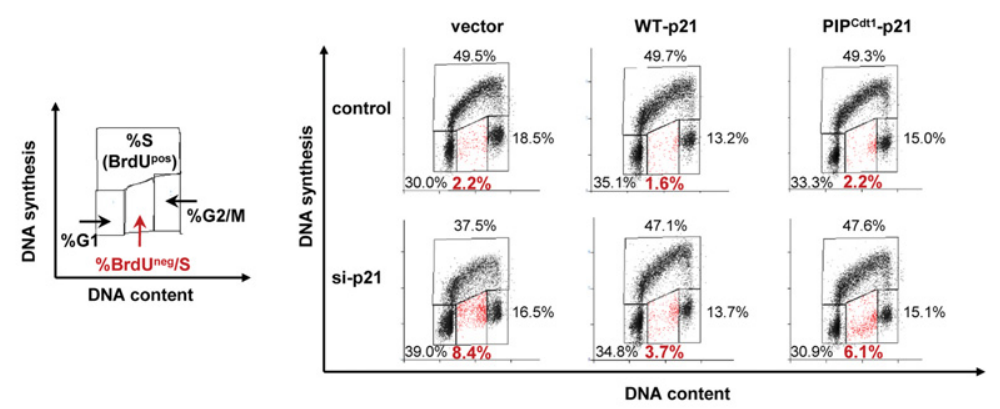

B

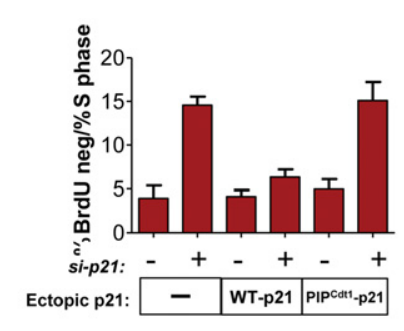

D

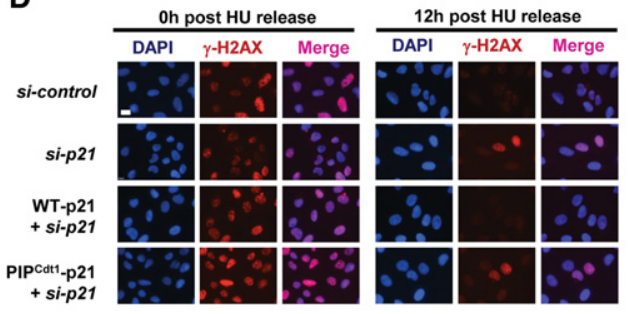

C

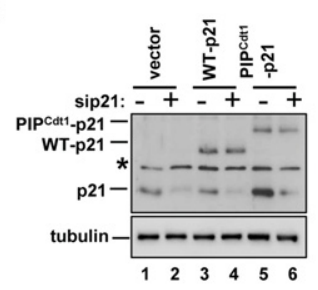

E

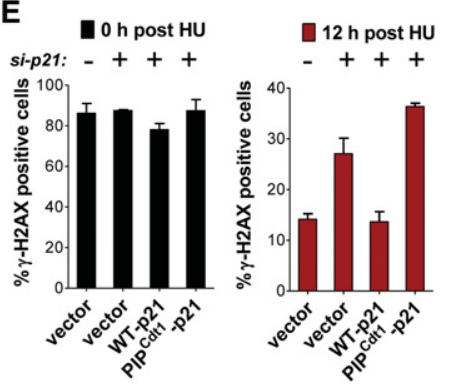

Figure 7. Accelerating $\mathrm{p} 21$ degradation promotes replication stress. (A) Asynchronously growing HCT116 cell lines stably expressing the indicated HA-tagged p21 fusions were treated with $100 \mathrm{nM}$ control or p21 siRNA for $48 \mathrm{~h}$. Cells were labeled for $1 \mathrm{~h}$ with BrdU and analyzed by flow cytometry with antiBrdU antibody to detect DNA synthesis (Y-axis) and with propidium iodide for DNA content ( $X$-axis). Flow cytometry gating for distinguishing BrdU-negative S-phase cells is indicated in the schematic. The percentage of the total population in each gated cell cycle phase is indicated on the plots. $(B)$ The percentage of BrdU-negative/S-phase cells in samples from $B$. Error bars indicate SEM. (C) Immunoblots of endogenous and ectopic p21 in $B .(D)$ U2OS cells producing the indicated $\mathrm{p} 21$-YFP fusions (induced with $10 \mathrm{ng} / \mathrm{mL}$ doxycycline) were treated with $2 \mathrm{mM}$ hydroxyurea $(\mathrm{HU})$ for $16 \mathrm{~h}$ and subsequently released into fresh medium for $12 \mathrm{~h}$, as indicated. $(D)$ Representative images show $\gamma-\mathrm{H} 2 \mathrm{AX}$ staining of cells for each condition. The percentage of $\gamma-\mathrm{H} 2 \mathrm{AX}$ positive cells is quantified in $E$. Bar, $30 \mu \mathrm{m}$. For quantifications, $>200$ nuclei from randomly selected fields were counted for each condition, and error bars represent standard deviations from two independent experiments. $P=<0.05$. of $\gamma$-H2AX foci (Fig. 7D,E). In contrast to both siRNA controls and p21-depleted cells complemented with wildtype p21, however, p21 depletion caused twofold more retention of $\gamma$-H2AX signal $12 \mathrm{~h}$ after HU release (Fig. 7D [12 h], E). Strikingly, cells expressing PIP ${ }^{\mathrm{Cdt} 1}$-p21 to levels similar to wild-type p21 (Supplemental Fig. 8A) retained as much $\gamma$-H2AX signal as p21-depleted cells did (Fig. 7C,D). Altogether, these results indicate that the normal delayed p21 degradation in early S phase contributes to robust replication progression during $\mathrm{S}$ phase. Furthermore, the absence of p21 during early $S$ phase sensitizes cells to subsequent replication stress and DNA damage.

\section{Discussion}

In this study, we provide conclusive evidence that, despite being subject to the same targeting mechanism, several substrates of the CRL4 ${ }^{\mathrm{Cdt} 2} \mathrm{E} 3$ ubiquitin ligase are degraded in a conserved and stereotypical order. In particular, p21 degradation is significantly delayed relative to Cdt1 (and PR-Set7) during both early S phase and DNA repair. As a result, early S-phase cells and cells beginning DNA repair lack Cdt1 but still have abundant $\mathrm{p} 21$. Several other studies (Bendjennat et al. 2003; Abbas et al. 2008, 2010; Nishitani et al. 2008; Shibata et al. 2011; Zhang et al. 2013) explored CRL4 ${ }^{\mathrm{Cdt2}}$ substrate degradation kinetics, and late 21 degradation is detectable in those studies but had not been investigated. We note also that these three proteins appear to reaccumulate at the end of $S$ phase in reverse order relative to the order in which they are degraded (Nishitani et al. 2008; Abbas et al. 2010; Rizzardi et al. 2014).

Based on our observations, early Cdt 1 degradation relative to p21 in S phase is likely due to differences in the efficiency with which different PIP degrons recruit Cdt2 (Fig. 8A). It is not yet clear, however, why the Cdt1 PIP degron confers weaker PCNA binding but more efficient $\mathrm{Cdt} 2$ recruitment. Detailed structural information about the interaction of p21's PIP degron with PCNA is available (Gulbis et al. 1996), and a collection of alanine substitutions at sites critical for PCNA binding or Cdt2 recruitment block replication-coupled destruction (Arias and Walter 2006; Havens and Walter 2009). Interestingly, however, none of those previously studied positions are significantly different between the p21 and Cdt1 PIP degrons (Supplemental Fig. 4B). We presume that the substantially more efficient in vitro Cdt 2 recruitment by the Cdt1 PIP degron is the major reason for faster Cdt1 degradation in vivo, but other mechanisms may also contribute. Among these factors are differences in ubiquitylation processivity, as is observed among substrates of the APC/C (Rape et al. 2006). Strikingly, p21's PIP degron binds PCNA ${ }^{\text {DNA }}$ in vitro more efficiently than Cdt1's PIP degron does (Fig. 6). It is possible that the p21- 
A

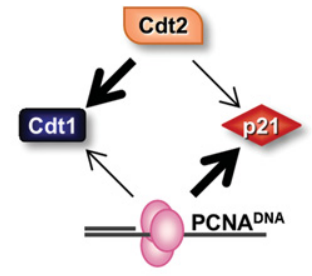

B

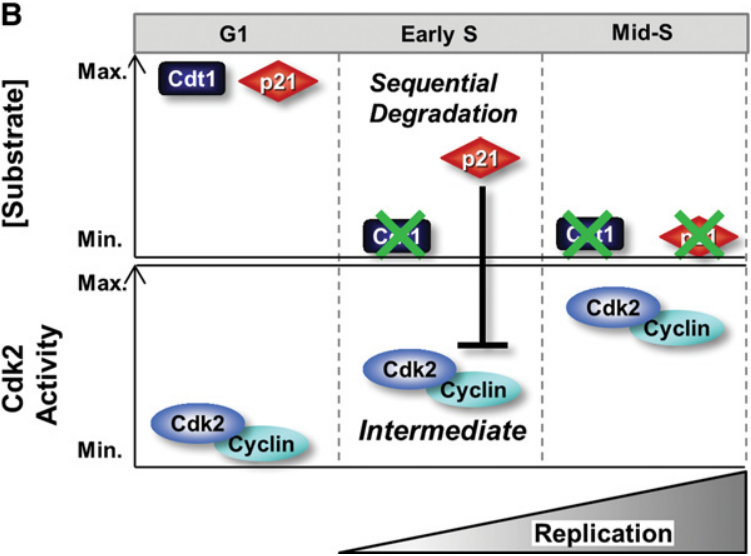

Figure 8. Model. (A)Despite lower binding affinity for PCNA, the Cdt1 PIP degron recruits more Cdt2 than the p21 PIP degron does. $(B)$ The normal relatively delayed degradation of $\mathrm{p} 21$ by CLR $4^{\mathrm{Cdt} 2}$ permits only intermediate Cdk2 activity in early $\mathrm{S}$ phase rather than maximal activity at S-phase onset. The more gradual degradation of p21 prevents replication stress (see the Discussion).

PCNA $^{\text {DNA }}$ interaction is in fact too tight for maximally efficient degradation compared with other CRL4 ${ }^{\mathrm{Cdt} 2}$ substrates, but this idea has not yet been explored. Tight PCNA binding by $\mathrm{p} 21$ has been suggested to compete with other PCNA-binding proteins (Warbrick et al. 1997; Ducoux et al. 2001; Arias and Walter 2006), and so we considered the possibility that competition among PIP degrons contributes to their order of degradation. However, we did not observe any evidence for competition effects either at the endogenous levels of CRL4 ${ }^{\text {Cdt2 }}$ substrates or when they are moderately overproduced. For example, the approximately twofold increase in PIP degrons from the ectopic fusions in Figure 4, A and B, had no effect on the rates of endogenous Cdt 1 and p 21 degradation. Thus, we do not favor a model in which the higher affinity of Cdt 2 for Cdt 1 leads to slower p21 degradation strictly through competition for either PCNA or Cdt2.

By fusing Cdt1's PIP degron to p21, we created a scenario in which p 21 is expressed in G1 but degraded prematurely at the beginning of $S$ phase rather than at the normal time well after S phase is under way. Interestingly, the consequences of accelerating the timing of $\mathrm{p} 21$ degradation are very similar to depleting cells of p21 altogether with respect to replication stress. The striking similarity of cells expressing the PIP ${ }^{\mathrm{Cdt}}$-p21 fusion to p21-depleted cells indicates that a critical period for $\mathrm{p} 21$ expression is early $\mathrm{S}$ phase, since PIP ${ }^{\text {Cdt1 }}$-p21 cells have sufficient p21 in G1. Cells lacking p21 in early S showed both an increase in the proportion of cells that failed $S$ phase and a reduced ability to recover from a strong replication block. Particularly with respect to the increase in $B r d U^{\text {neg }} / \mathrm{S}$-phase cells, we were initially surprised to observe what appeared to be a mid-to-late S-phase phenotype, since mid-S-phase cells have already degraded $\mathrm{p} 21$. In addition, the recovery from an HU block seems unrelated to the degradation rates of the p21 fusions during the arrest, since both are degraded at $0 \mathrm{~h}$. However, we consider that these phenotypes are a downstream consequence of insufficient p21 in early S phase, leading to overall higher levels of endogenous damage prior to or during the HU block and a longer recovery time. Even in the absence of HU treatment, we observed a small (although not statistically significant) increase in $\gamma$-H2AX-positive proliferating cells expressing only the prematurely degraded $\mathrm{p} 21$ (data not shown). The combination of pre-existing replication stress with an HU challenge could account for the poor recovery from replication arrest.

Our findings suggest a model for the need to degrade $\mathrm{Cdt} 1$ at the very beginning of $\mathrm{S}$ phase but delay $\mathrm{p} 21$ degradation until later in S phase (Fig. 8B). In normal cells, Cdk2 activity begins to rise in late G1 due to the increase in cyclin E, but p21 is still present (Fig. 1). Once S phase begins and PCNA is loaded at early-firing origins, the continued presence of $\mathrm{p} 21$ restrains S-phase progression by slowing the rise of Cdk2 activity. In this model, early S-phase cells have lower total Cdk2 activity than mid-S cells, but the intermediate Cdk2 activity supports some origin firing and PCNA loading. During this early S-phase period, Cdt1 is rapidly degraded to prevent origin relicensing and rereplication. This rapid degradation may be particularly important because two of the blocks to rereplication, the geminin inhibitor protein and cyclin A, are lower in early $S$ phase than when they reach maximal levels later in S (Girard et al. 1991; Rosenblatt et al. 1992; Wohlschlegel et al. 2000). If $\mathrm{p} 21$ is degraded prematurely, Cdk 2 activity may rise too early, leading to more origins firing at the beginning of S phase than normally should and subsequent replication stress. In support of that notion, manipulations in both yeast and Xenopus laevis experimental systems that shift origin firing towards early $S$ phase induce replication stress markers (Gibson et al. 2004; Woodward et al. 2006; Mantiero et al. 2011). Replication fork speed may also be affected by p21 tightly bound to PCNA (Waga et al. 1994). The aggregate of effects such as these may explain the higher likelihood of S-phase failure in cells that prematurely degrade $\mathrm{p} 21$. We therefore conclude that some key molecular events at the G1/S transition must happen in a defined order to ensure a normal S-phase progression. These findings raise the possibility that many aspects of cell cycle transitions are also programmed to occur in a stereotypical sequence to ensure genome stability.

\section{Materials and methods}

\section{Cell culture and manipulations}

HCT116 and HEK 293T cells were obtained from American Type Culture Collection and cultured in Dulbecco's modified Eagle's medium (DMEM) (Sigma) supplemented with 10\% fetal calf 
serum (Sigma). U2OS TRex cells were a gift from John Aster (Malecki et al. 2006). NHF-hTert cells are normal human fibroblasts immortalized with telomerase. HCT116 cells were synchronized in prometaphase by treatment with $2 \mathrm{mM}$ thymidine for $18 \mathrm{~h}$ followed by release into $100 \mathrm{nM}$ nocodazole. To obtain populations of cells in either G1 or early S phase, cells were released from the prometaphase arrest by mitotic shake-off, replated in complete medium, and collected at either $2.5 \mathrm{~h}$ (G1) or $4 \mathrm{~h}$ (early S phase). To arrest replication forks and measure recovery, U2OS cells expressing empty vector, wild-type p21, or PIP ${ }^{\mathrm{Cdt1}}$-p21 were treated with $2 \mathrm{mM}$ HU for $16 \mathrm{~h}$ and released into fresh medium. UV irradiation experiments were performed using a single dose of $20 \mathrm{~J} / \mathrm{m}^{2}$ in a Stratalinker (Stratagene). Additional DNA damage repair triggers used were $100 \mu \mathrm{M}$ tert-butyl peroxide (Sigma) and $10 \mu \mathrm{g} / \mathrm{mL}$ bleocin (EMD Millipore). Lentiviral packaging was performed by standard protocols in $293 \mathrm{~T}$ cells followed by infection and selection of HCT116 cells with $1 \mu \mathrm{g} / \mathrm{mL}$ puromycin. Synthetic duplexed RNA oligonucleotides were synthesized by Life Technologies: Luciferase (5'-CUUACGCUGAGUACUUCGA-3'), p21-2 (5'-AACAUACUGGCCUGGACUGUU-3'), UBCH8 (5'-GC AAGAACCAGAAAGAGAA-3'), UBE2G1 (5'-GGGAAGAUAAG UAUGGUUA-3'), and UBE2G2 (5'-UGACGAAAGUGGAGCUA AC- $3^{\prime}$ ). Significance testing used the two-tailed Student's $t$-test on at least three biological replicates.

\section{Antibodies}

Antibodies were purchased from the following sources: p21, PCNA, and anti-HA from Santa Cruz Biotechnology; PR-Set7 and Cdt1 from Cell Signaling Technologies; $\alpha$-tubulin, GAPDH, and GST from Sigma; anti-BrdU from BD Biosciences; anti-phospho-Histone H2A.X (Ser139) from EMD Millipore; anti-HA from Roche Life Sciences; anti-UBCH8 and anti-UBE2G1 from Protein Tech; and anti-UBE2G2 from Thermo Scientific. Antibodies to human Cdt1 (Cook et al. 2004) and Cdt2 (Abbas et al. 2008) have been described previously. Alexa fluor 488, Rhodamine Red-X, and Cy 5 donkey secondary antibodies for immunofluorescence microscopy were obtained from Jackson ImmunoResearch Laboratories.

\section{Plasmids and recombinant protein preparation}

For expression in mammalian cells, wild-type p21, PIP ${ }^{\mathrm{Cdt} 1}$-p21, PIP ${ }^{\mathrm{Cdt} 1}$-GST, PIP ${ }^{\mathrm{p} 21}$-GST, wild-type Cdt1 bearing an HA epitope tag, Cdt1 15-546-HA (" $\Delta \mathrm{PIP}^{\prime \prime}$ ), and PIP ${ }^{\mathrm{p} 21}$-Cdt1-HA alleles were subcloned into pENTR vectors and subsequently transferred into a retroviral vector, pQCXIP CMV TO/DEST (Addgene, 17386) (Campeau et al. 2009), to pDESTJC16 or pDESTJC17 by Gateway cloning. The K153A mutation in p21 was generated through site-directed mutagenesis. Lentiviral PIPm ${ }^{\mathrm{Cdt1}}$-p21, PIP ${ }^{\mathrm{Cdt} 1}$-p21PIPm, PIP ${ }^{\mathrm{p} 21}$-p21, and PIPm-p21 plasmids were generated by subcloning cDNAs into a pCDH IRES mcherry vector (System Biosciences). pBabe-puro-H2B-mCherry was subcloned from PGK-H2B-mCherry (gift from Mark Mercola; Addgene, plasmid no. 21217) into pBabe-puro using standard methods. pDESTJC16 and pDESTJC17 are derivatives of pcDNA5/FRT/ TO-Venus-Flag-Gateway (gift from Jonathon Pines; Addgene, plasmid 40999) in which the hygromycin resistance gene has been replaced with either the puromycin or the nourseothricin resistance gene, respectively, using standard methods. pEXPJC17-Cdt1-mCerulean3-P2A-Venus-NLS-p21, pEXP-JC17-2HNVenus, and pEXP-JC17-Venus-NLS-p21 were generated by Gateway cloning from pENTR vectors. Both Venus and mCerulean3 fluorophores were selected for their relative brightness and rapid fluorescence maturation. Plasmids for expressing wild-type and
PIPm-p21 GST fusions were generated by recombinational Gateway cloning (LR Clonase, Invitrogen) between pENTR plasmids and pDEST15. Additional plasmids for PIP ${ }^{\mathrm{Cdt1}}{ }_{-}$GST and PIP ${ }^{\mathrm{p} 21}$ GST fusions were constructed by subcloning cDNAs into pET11b vector (Novagen). The PIP mutants were generated by site-directed mutagenesis to introduce alanine codons a positions corresponding to Cdt 1 amino acids 5-8 or p21 amino acids 146150. PIP degron fusions carried human Cdt 1 amino acids $1-28$ or human p21 amino acids 137-164. GST fusions were transformed into BL21 (DE3) cells (Invitrogen) and partially purified as described previously (Cook et al. 2004).

\section{Protein lysate preparation}

Whole-cell lysates were prepared by direct lysis of equal cell numbers in either $2 \times$ Laemmli sample buffer with $10 \% \beta$-mercaptoethanol or CSK buffer (Cook et al. 2002) supplemented with $0.1 \%$ Triton X-100 and protease and phosphatase inhibitors. Densitometry analysis of immunoblots of lysates was performed using $\mathrm{Na}$ tional Institutes of Health ImageJ software (normalized to tubulin expression). At least two film exposures were chosen for quantifications of each protein analyzed such that one lane of each exposure was of equal band intensity and thereby served as a normalization control. Quantifications represent percentages of each protein remaining relative to the starting amount prior to $\mathrm{UV}$ irradiation (time $=0 \mathrm{~min}$ ). Half-lives were estimated from semi-log plots of the $\ln$ (percentage remaining) versus time.

\section{GST pull-down assays}

The recombinant GST fusion proteins described above were immobilized on glutathione-sepharose 4B resin (GE Healthcare). UV-irradiated HEK293T cells were lysed in CSK buffer (supplemented with $0.1 \%$ Triton X-100 plus protease and phosphatase inhibitors). Chromatin fractions were prepared as described previously (Cook et al. 2002) and subjected to low-power sonication of Triton-insoluble pellets to fragment DNA-bound material. Soluble chromatin fractions were clarified by centrifugation at $13,000 \mathrm{~g}$ for $15 \mathrm{~min}$ at $4^{\circ} \mathrm{C}$ and incubated with bound GST fusion proteins for $3 \mathrm{~h}$ with rotation at $4^{\circ} \mathrm{C}$. Beads were washed three times in supplemented CSK buffer, and bound proteins were eluted by boiling for $5 \mathrm{~min}$ in $40 \mu \mathrm{L}$ of $2 \times$ SDS sample buffer.

\section{Immunofluorescence microscopy}

HCT116 cells synchronized in either G1 or early S phase were pulse-labeled with $50 \mu \mathrm{M}$ BrdU for $30 \mathrm{~min}$ prior to fixation in $2 \%$ paraformaldehyde for $15 \mathrm{~min}$. DNA was denatured with $1.5 \mathrm{~N}$ hydrochloric acid for $15 \mathrm{~min}$. Cells were permeabilized with $0.5 \%$ Triton-X-100 for $5 \mathrm{~min}$ and then blocked in $0.1 \%$ BSA for $1 \mathrm{~h}$. Incubations with primary and fluorescent secondary antibodies were performed in $0.1 \%$ BSA for $1 \mathrm{~h}$ at $37^{\circ} \mathrm{C}$, and washes were for $10 \mathrm{~min}$ at room temperature. DAPI $\left(4^{\prime}, 6\right.$-diamidino-2phenylindole) staining $(0.1 \mu \mathrm{g} / \mathrm{mL})$ was performed for $10 \mathrm{~min}$, and cells were mounted using Prolong Gold anti-fade reagent (Life Technologies/Molecular Probes).

For indirect immunofluorescence microscopy, images were acquired using Velocity software and a 60×/1.42 NA (PlanApo) DIC oil immersion objective mounted on a Olympus 1 X81 inverted microscope equipped with a Hamamatsu Orca ER cooled CCD camera. For fluorescence intensity measurements, the average values for integrated nuclear Cdt 1 and $\mathrm{p} 21$ fluorescence from control and experimental cell samples were subjected to background subtraction to obtain the specific nuclear fluorescence levels. 


\section{Flow cytometry analysis}

Cells to be analyzed by flow cytometry were labeled with $10 \mu \mathrm{M}$ BrdU for $1 \mathrm{~h}$ prior to trypsinization and fixation in $70 \%$ ethanol. Nuclei were stained with anti-BrdU antibody (BD Biosciences) followed by Alexa fluor 488-labeled secondary antibody (Jackson ImmunoResearch Laboratories) and were counterstained with propidium iodide. Flow cytometry analysis was performed using a Cyan FACScan (DakoCytomation) and Summit version 4.3 software (DakoCytomation).

\section{Fixed-cell imaging analysis}

Asynchronous U2OS cells expressing Cdt1-mCerulean3-P2AVenus-p21 induced with $20 \mathrm{ng} / \mathrm{mL}$ doxycycline were fixed with $2 \%$ paraformaldehyde using standard methods and imaged on a Nikon Ti Eclipse inverted microscope running NIS Elements software version 4.30.02 with an Andor ZYLA 4.2 camera using a $20 \times$ objective. Images of randomly selected fields were collected in all three channels (CFP, YFP, and RFP) using exposure times producing approximately equivalent nuclear fluorescence in the three channels. Image (version $1.49 \mathrm{p}$ ) was used to identify nuclei on the H2B-mCherry images using the nucleus counter plug-in from the ImageJ Cookbook. Cells were scored for Venus and/or mCerulean3 expression over three randomly selected fields ( $n>150$ per field).

\section{Live-cell imaging analysis}

Asynchronous U2OS cells stably expressing histone H2BmCherry and PIP ${ }^{C d t 1}$-p21-Venus or Venus-NLS-p21 were plated on 35-mm glass-bottom plates (Cellvis) in imaging medium (phenol-red-free DMEM [Invitrogen] supplemented with 10\% FBS and $1 \times$ pennicillin/streptomycin) $\sim 24 \mathrm{~h}$ before being imaged. Cells were imaged for a minimum of $18 \mathrm{~h}$ using a Nikon Ti Eclipse microscope operated by NIS Elements software version 4.30.02 with an Andor ZYLA 4.2 camera and a custom stage enclosure to ensure constant temperature, humidity, and $\mathrm{CO}_{2}$ levels. Protein expression was induced with $10 \mathrm{ng} / \mathrm{mL}$ (PIP ${ }^{\mathrm{Cdt1}}-\mathrm{p} 21$ ), or $30 \mathrm{ng} / \mathrm{mL}$ (Ven-NLS-p21) doxycycline $20 \mathrm{~h}$ before imaging. Fresh medium with doxycycline was added $5 \mathrm{~h}$ before imaging. Images were flat-field-corrected using NIS Elements. Fifty cells of each line were selected for analysis based on Venus expression and visualization of a complete cycle (minimum mitosis to S-phase degradation).

Single-cell fluorescence intensity was quantified using custom MATLAB software, which is available on request. In each frame, the nuclei of individual cells were identified using an adaptive threshold followed by watershed segmentation of the H2BmCherry channel. Cells were tracked through consecutive frames by tracking nearest nuclear centroids between frames. The resulting nuclear borders were used to quantify average fluorescence intensity from other channels (e.g., p21-Venus). Missing time points, which were generally no more than one to two frames, were interpolated linearly. To compare the decay kinetics of individual cells, fluorescence intensity was normalized between 0 and 1 . The delay time, $T_{\mathrm{d}}$, was calculated by fitting each trace to an inverse sigmoidal Boltzmann curve:

$$
f(t)=\frac{1}{1+\exp \left[\left(t-t_{d}\right) / t_{s}\right]} .
$$

\section{Acknowledgments}

We are grateful to J. Aster (Brigham and Women's Hospital) for the gift of U2OS-TRex cells; S. Wolff, J. Jones, D. Tesfu, P. Pozo,
J. Matson, S. Oh, and S. Aslam for technical and general assistance during the course of this work; and M. Emanuele and members of the Cook laboratory for comments on the manuscript. The University of North Carolina Flow Cytometry Core Facility is supported in part by a National Cancer Institute Center Core Support Grant (P30CA016086) to the University of North Carolina Lineberger Comprehensive Cancer Center. The Microscopy Services Laboratory is supported by the University of North Carolina Department of Pathology and Laboratory Medicine and the Lineberger Comprehensive Cancer Center. This work was supported by National Institutes of Health National Institute of General Medical Sciences grants R01GM102413 (to J.G.C.) and R00GM102372 (to J.E.P.) and National Institutes of Health National Cancer Institute grants K99CA178177 (to D.V.), F31CA165891 (to K.E.C.), and T32CA009156 (to G.D.G.).

\section{References}

Abbas T, Sivaprasad U, Terai K, Amador V, Pagano M, Dutta A. 2008. PCNA-dependent regulation of p21 ubiquitylation and degradation via the CRL4Cdt2 ubiquitin ligase complex. Genes Dev 22: 2496-2506.

Abbas T, Shibata E, Park J, Jha S, Karnani N, Dutta A. 2010. CRL4 (Cdt2) regulates cell proliferation and histone gene expression by targeting PR-Set7/Set8 for degradation. Mol Cell 40: 9-21.

Arias EE, Walter JC. 2005. Replication-dependent destruction of Cdt1 limits DNA replication to a single round per cell cycle in Xenopus egg extracts. Genes Dev 19: 114-126.

Arias EE, Walter JC. 2006. PCNA functions as a molecular platform to trigger Cdt1 destruction and prevent re-replication. Nat Cell Biol 8: 84-90.

Bendjennat M, Boulaire J, Jascur T, Brickner H, Barbier V, Sarasin A, Fotedar A, Fotedar R. 2003. UV irradiation triggers ubiquitin-dependent degradation of $\mathrm{p} 21$ (WAF1) to promote DNA repair. Cell 114: 599-610.

Campeau E, Ruhl VE, Rodier F, Smith CL, Rahmberg BL, Fuss JO, Campisi I, Yaswen P, Cooper PK, Kaufman PD. 2009. A versatile viral system for expression and depletion of proteins in mammalian cells. PLoS One 4: e6529.

Centore RC, Havens CG, Manning AL, Li J-M, Flynn RL, Tse A, Jin J, Dyson NJ, Walter JC, Zou L. 2010. CRL4 ${ }^{\text {Cdt2 }}$-mediated destruction of the histone methyltransferase Set8 prevents premature chromatin compaction in S Phase. Mol Cell 40: 22-33.

Chandrasekaran S, Tan TX, Hall JR, Cook JG. 2011. Stress-activated MAP kinases, p38 and JNK, control the stability and activity of the Cdt1 DNA replication licensing factor. Mol Cell Biol 31: 4405-4416.

Cook JG, Park C-H, Burke TW, Leone G, DeGregori J, Engel A, Nevins JR. 2002. Analysis of Cdc6 function in the assembly of mammalian prereplication complexes. Proc Nat1 Acad Sci 99: $1347-1352$.

Cook JG, Chasse DA, Nevins JR. 2004. The regulated association of Cdt1 with minichromosome maintenance proteins and Cdc6 in mammalian cells. J Biol Chem 279: 9625-9633.

den Elzen N, Pines J. 2001. Cyclin A is destroyed in prometaphase and can delay chromosome alignment and anaphase. J Cell Biol 153: 121-136.

Ducoux M, Urbach S, Baldacci G, Hübscher U, Koundrioukoff S, Christensen J, Hughes P. 2001. Mediation of proliferating cell nuclear antigen (PCNA)-dependent DNA replication through a conserved p21(Cip1)-like PCNA-binding motif present in the third subunit of human DNA polymerase $\delta$. J Biol Chem 276: 49258-49266.

Gibson DG, Aparicio JG, Hu F, Aparicio OM. 2004. Diminished S-phase cyclin-dependent kinase function elicits vital 
Rad53-dependent checkpoint responses in Saccharomyces cerevisiae. Mol Cell Biol 24: 10208-10222.

Girard F, Strausfeld U, Fernandez A, Lamb NJ. 1991. Cyclin A is required for the onset of DNA replication in mammalian fibroblasts. Cell 67: 1169-1179.

Gulbis JM, Kelman Z, Hurwitz J, Donnell MO, Kuriyan J. 1996. Structure of the C-terminal region complexed with human PCNA. Cell 87: 297-306.

Hagting A, Den Elzen N, Vodermaier HC, Waizenegger IC, Peters J-M, Pines J. 2002. Human securin proteolysis is controlled by the spindle checkpoint and reveals when the APC/C switches from activation by Cdc20 to Cdh1. J Cell Biol 157: 1125-1137.

Han C, Wani G, Zhao R, Qian J, Sharma N, He J, Zhu Q, Wang Q-E, Wani AA. 2014. Cdt2-mediated XPG degradation promotes gap-filling DNA synthesis in nucleotide excision repair. Cell Cycle 14: 1103-1115.

Havens CG, Walter JC. 2009. Docking of a specialized PIP Box onto chromatin-bound PCNA creates a degron for the ubiquitin ligase CRL4Cdt2. Mol Cell 35: 93-104.

Havens CG, Shobnam N, Guarino E, Centore RC, Zou L, Kearsey SE, Walter JC. 2012. Direct role for proliferating cell nuclear antigen in substrate recognition by the E3 ubiquitin ligase CRL4Cdt2. J Biol Chem 287: 11410-11421.

Izawa D, Pines J. 2011. How APC/C-Cdc20 changes its substrate specificity in mitosis. Nat Cell Biol 13: 223-233.

Jørgensen S, Eskildsen M, Fugger K, Hansen L, Larsen MSY, Kousholt AN, Syljuåsen RG, Trelle MB, Jensen ON, Helin $\mathrm{K}$, et al. 2011. SET8 is degraded via PCNA-coupled CRL4 (CDT2) ubiquitylation in S phase and after UV irradiation. $J$ Cell Biol 192: 43-54.

Kim JH, Lee SR, Li LH, Park HJ, Park JH, Lee KY, Kim MK, Shin BA, Choi SY. 2011. High cleavage efficiency of a 2A peptide derived from porcine teschovirus- 1 in human cell lines, zebrafish and mice. PLoS One 6: 1-8.

Lindon C, Pines J. 2004. Ordered proteolysis in anaphase inactivates Plk1 to contribute to proper mitotic exit in human cells. J Cell Biol 164: 233-241.

Lu D, Hsiao JY, Davey NE, Van Voorhis VA, Foster SA, Tang C, Morgan DO. 2014. Multiple mechanisms determine the order of APC/C substrate degradation in mitosis. I Cell Biol 207: 23-39.

Malecki MJ, Sanchez-Irizarry C, Mitchell JL, Histen G, Xu ML, Aster JC, Blacklow SC. 2006. Leukemia-associated mutations within the NOTCH1 heterodimerization domain fall into at least two distinct mechanistic classes. Mol Cell Biol 26: 4642-4651.

Mantiero D, Mackenzie A, Donaldson A, Zegerman P. 2011. Limiting replication initiation factors execute the temporal programme of origin firing in budding yeast. $E M B O J$ 30: $4805-4814$.

Nakayama KI, Nakayama K. 2006. Ubiquitin ligases: cell-cycle control and cancer. Nat Rev Cancer 6: 369-381.

Nishitani H, Sugimoto N, Roukos V, Nakanishi Y, Saijo M, Obuse C, Tsurimoto T, Nakayama KI, Nakayama K, Fujita M, et al. 2006. Two E3 ubiquitin ligases, SCF-Skp2 and DDB1-Cul4, target human Cdt1 for proteolysis. EMBO I 25: 1126-1136.

Nishitani H, Shiomi Y, Iida H, Michishita M, Takami T, Tsurimoto T. 2008. CDK inhibitor 21 is degraded by a proliferating cell nuclear antigen-coupled Cul4-DDB1Cdt2 pathway during $S$ phase and after UV irradiation. I Biol Chem 283: 29045-29052.

Oda H, Hübner MR, Beck DB, Vermeulen M, Hurwitz J, Spector DL, Reinberg D. 2010. Regulation of the histone H4 monomethylase PR-Set7 by CRL4(Cdt2)-mediated PCNA-dependent degradation during DNA damage. Mol Cell 40: 364-376.
Pagano M, Theodoras AM, Tam SW, Draetta GF. 1994. Cyclin D 1-mediated inhibition of repair and replicative DNA synthesis in human fibroblasts. Genes Dev 8: 1627-1639.

Petroski MD, Deshaies RJ. 2005. Function and regulation of cullin-RING ubiquitin ligases. Nat Rev Mol Cell Biol 6: 9-20.

Rape M, Reddy SK, Kirschner MW. 2006. The processivity of multiubiquitination by the APC determines the order of substrate degradation. Cell 124: 89-103.

Rizzardi LF, Coleman KE, Varma D, Matson JP, Oh S, Cook JG. 2014. Cyclin-dependent kinase 1 (CDK1)-dependent inhibition of the E3 ubiquitin ligase, CRL4CDT2, ensures robust transition from S Phase to Mitosis. J Biol Chem 290: 556-567.

Rosenblatt J, Gu Y, Morgan DO. 1992. Human cyclin-dependent kinase 2 is activated during the $S$ and G2 phases of the cell cycle and associates with cyclin A. Proc Nat1 Acad Sci 89:2824-2828.

Senga T, Sivaprasad U, Zhu W, Park JH, Arias EE, Walter JC, Dutta A. 2006. PCNA is a cofactor for Cdt1 degradation by CUL4/DDB1-mediated N-terminal ubiquitination. I Biol Chem 281: 6246-6252.

Shibata E, Abbas T, Huang X, Wohlschlegel JA, Dutta A. 2011. Selective ubiquitylation of $\mathrm{p} 21$ and $\mathrm{Cdt} 1$ by UBCH8 and UBE2G ubiquitin-conjugating enzymes via the CRL4Cdt2 ubiquitin ligase complex. Mol Cell Biol 31: 3136-3145.

Shibata E, Dar A, Dutta A. 2014. CRL4Cdt2 E3 ubiquitin ligase and PCNA cooperate to degrade thymine DNA glycosylase in S-phase. J Biol Chem 289: 23056-23064.

Slenn TJ, Morris B, Havens CG, Freeman RM, Takahashi TS, Walter JC. 2014. Thymine DNA glycosylase is a CRL4Cdt2 substrate. J Biol Chem 289: 23043-23055.

Tardat M, Brustel J, Kirsh O, Lefevbre C, Callanan M, Sardet C, Julien E. 2010. The histone H4 Lys 20 methyltransferase PRSet7 regulates replication origins in mammalian cells. Nat Cell Biol 12: 1086-1093.

Teixeira LK, Reed SI. 2013. Ubiquitin ligases and cell cycle control. Annu Rev Biochem 82: 387-414.

Terai K, Shibata E, Abbas T, Dutta A. 2013. Degradation of p12 subunit by CRL4Cdt2 E3 ligase inhibits fork progression after DNA damage. J Biol Chem 288: 30509-30514.

Waga S, Hannon GJ, Beach D, Stillman B. 1994. The p21 inhibitor of cyclin-dependent kinases controls DNA replication by interaction with PCNA. Nature 369: 574-578.

Warbrick E, Lane DP, Glover DM, Cox LS. 1997. Homologous regions of Fen1 and p21Cipl compete for binding to the same site on PCNA: a potential mechanism to co-ordinate DNA replication and repair. Oncogene 14: 2313-2321.

Wohlschlegel JA, Dwyer BT, Dhar SK, Cvetic C, Walter JC, Dutta A. 2000. Inhibition of eukaryotic DNA replication by geminin binding to Cdt1. Science 290: 2309-2312.

Woodward AM, Göhler T, Luciani MG, Oehlmann M, Ge X, Gartner A, Jackson DA, Blow JJ. 2006. Excess Mcm2-7 license dormant origins of replication that can be used under conditions of replicative stress. J Cell Biol 173: 673-683.

Zhang L, Mei Y, Fu N-Y, Guan L, Xie W, Liu H-H, Yu C-D, Yin Z, Yu VC, You H. 2012. TRIM39 regulates cell cycle progression and DNA damage responses via stabilizing p21. Proc Nat1 Acad Sci 109: 20937-20942.

Zhang S, Zhao H, Darzynkiewicz Z, Zhou P, Zhang Z, Lee EYC, Lee MYWT. 2013. A novel function of CRL4(Cdt2): regulation of the subunit structure of DNA polymerase $\delta$ in response to DNA damage and during the S phase. J Biol Chem 288: 29550-29561.

Zhao H, Zhang S, Xu D, Lee MY, Zhang Z, Lee EY, Darzynkiewicz Z. 2014. Expression of the p12 subunit of human DNA polymerase $\delta(\mathrm{Pol} \delta)$, CDK inhibitor p21(WAF1), Cdt1, cyclin A, PCNA and Ki-67 in relation to DNA replication in individual cells. Cell Cycle 13: 3529-3540. 


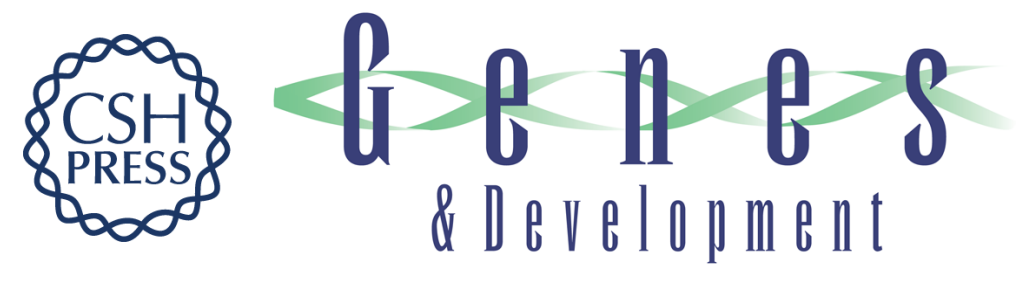

\title{
Sequential replication-coupled destruction at G1/S ensures genome stability
}

\author{
Kate E. Coleman, Gavin D. Grant, Rachel A. Haggerty, et al.
}

Genes Dev. 2015, 29: originally published online August 13, 2015

Access the most recent version at doi:10.1101/gad.263731.115

\section{Supplemental http://genesdev.cshlp.org/content/suppl/2015/08/12/gad.263731.115.DC1 Material}

References This article cites 49 articles, 28 of which can be accessed free at: http://genesdev.cshlp.org/content/29/16/1734.full.html\#ref-list-1

Creative This article is distributed exclusively by Cold Spring Harbor Laboratory Press for the first Commons six months after the full-issue publication date (see

License http://genesdev.cshlp.org/site/misc/terms.xhtml). After six months, it is available under a Creative Commons License (Attribution-NonCommercial 4.0 International), as described at http://creativecommons.org/licenses/by-nc/4.0/. Email Alerting
Service

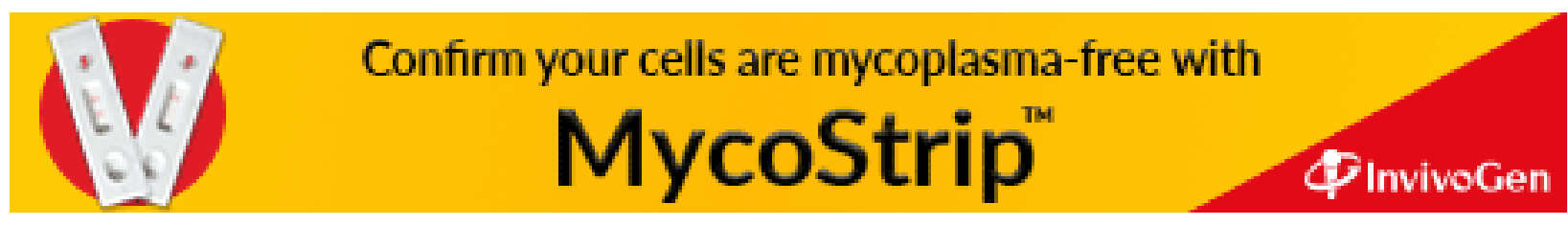

